\title{
Testing electroweak SUSY for muon $g-2$ and dark matter at the LHC and beyond
}

\author{
Murat Abdughani, ${ }^{a, b, c}$ Ken-ichi Hikasa, ${ }^{d}$ Lei Wu, ${ }^{c}$ Jin Min Yang ${ }^{a, b}$ and Jun Zhao ${ }^{a, b}$ \\ ${ }^{a}$ CAS Key Laboratory of Theoretical Physics, Institute of Theoretical Physics, \\ Chinese Academy of Sciences, \\ Beijing 100190, P.R. China \\ ${ }^{b}$ School of Physical Sciences, University of Chinese Academy of Sciences, \\ Beijing 100049, P.R. China \\ ${ }^{c}$ Department of Physics and Institute of Theoretical Physics, Nanjing Normal University, \\ Nanjing 210023, P.R. China \\ ${ }^{d}$ Department of Physics, Tohoku University, \\ Sendai 980-8578, Japan \\ E-mail: mulati@itp.ac.cn, hikasa@phys.tohoku.ac.jp, leiwu@itp.ac.cn, \\ jmyang@itp.ac.cn, zhaojun@itp.ac.cn
}

ABSTRACT: Given that the LHC experiment has produced strong constraints on the colored supersymmetric particles (sparticles), testing the electroweak supersymmetry (EWSUSY) will be the next crucial task at the LHC. On the other hand, the light electroweakinos and sleptons in the EWSUSY can also contribute to the dark matter (DM) and low energy lepton observables. The precision measurements of them will provide the indirect evidence of SUSY. In this work, we confront the EWSUSY with the muon $g-2$ anomaly, the DM relic density, the direct detection limits and the latest LHC Run-2 data. We find that the sneutrino DM or the neutralino DM with sizable higgsino component has been excluded by the direct detections. Then two viable scenarios are pinned down: one has the light compressed bino and sleptons but heavy higgsinos, and the other has the light compressed bino, winos and sleptons. In the former case, the LSP and slepton masses have to be smaller than about $350 \mathrm{GeV}$. While in the latter case, the LSP and slepton masses have to be smaller than about $700 \mathrm{GeV}$ and $800 \mathrm{GeV}$, respectively. From investigating the observability of these sparticles in both scenarios at future colliders, it turns out that the HE-LHC with a luminosity of $15 \mathrm{ab}^{-1}$ can exclude the whole BHL and most part of BWL scenarios at $2 \sigma$ level. The precision measurement of the Higgs couplings at the lepton colliders could play a complementary role of probing the BWL scenario.

KEYWORDs: Supersymmetry Phenomenology

ARXIV EPRINT: 1909.07792 


\section{Contents}

1 Introduction 1

2 neutralino dark matter and muon $g-2$ in the MSSM 2

3 Parameter scan for muon $g-2$ and dark matter 4

4 observabilities at LHC upgrades and Higgs factory 6

5 Conclusions 12

\section{Introduction}

In particle physics, the muon anomalous magnetic moment $a_{\mu}$ is one of the most precisely measured quantities. Since the first results were reported, there has been a longstanding $\sim 3 \sigma$ discrepancy between theory and experiment, which triggered numerous studies of new physics explanations. As a successor of the previous E821 experiment performed at BNL, the on-going muon $g-2$ experiment E989 at Fermilab is to measure $a_{\mu}$ with a relative precision of 140 parts-per-billion (ppb) [1]. This precision is a factor of four improvement from the current experiment [2]. If this anomaly still persists, it would be a clear evidence for new physics beyond the Standard Model (BSM).

Meanwhile, dark matter (DM) that constitutes the majority of matter in the universe has been established by astrophysical and cosmological observations. Understanding its nature and interactions is one of the most important quests of contemporary physics. The Weakly Interacting Massive Particle (WIMP) paradigm provides an attractive solution to the DM issue as it can naturally produce the measured relic density through the robust mechanism of thermal freeze-out. Therefore, various concrete realizations of WIMP models have been proposed, which has been being tested in DM (in)direct detections and collider experiments [3].

Among new physics models for solving these two problems, supersymmetry (SUSY) is one of the most popular candidates, which has a beautiful mathematical structure and is considered as a part of a larger vision of physics. In supersymmetric models, the lightest neutralino $\tilde{\chi}_{1}^{0}$ can serve as a natural WIMP DM candidate if the $R$-parity is conserved. Meanwhile, the muon $g-2$ anomaly can be explained by the contributions of light sleptons and electroweakinos running in the loops [4-7]. In addition, SUSY can also solve the hierarchy problem and realize the unification of gauge couplings at the GUT scale. Due to its overwhelming virtues and popularity, the low energy SUSY has long been pursued by both theorists and experimentalists.

Up to now, the LHC null observation of colored sparticles has excluded the masses of squarks and gluinos lighter than about $2 \mathrm{TeV}$ in simplified models [8]. Fortunately, to 
account for the DM abundance and the muon $g-2$ anomaly, only the uncolored sparticles (electroweakinos and sleptons) need to be light, which are subject to relatively rather weak constraints from the LHC searches [9]. As a result, the electroweak SUSY that only consists of light electroweakinos and sleptons is strongly favored by current experimental data. As shown in refs. [10-28], such a scenario can be realized in well-motivated high scale supersymmetric models.

In this work we perform a comprehensive study of the phenomenology of the EWSUSY scenario for the muon $g-2$ and dark matter. Note that in the literature [29-36] such a scenario has been discussed to some extent. However, those studies either did not require the SUSY dark matter to provide the correct abundance or focused on the phenomenology at the LHC. Unlike them, we pin down the viable parameter space of EWSUSY for explaining the dark matter abundance and the muon $g-2$ anomaly by a numerical scan. We find that the masses of the electroweakinos and sleptons are bounded in certain ranges, which will guide the search strategies at colliders. In addition to the LHC observability of such a scenario, we will also explore its test at the LHC upgrades and the $e^{+} e^{-}$Higgs factory. As a precison test machine, the future $e^{+} e^{-}$Higgs factories, such as CEPC, FCCee and ILC, have limited energy to directly search for SUSY particles. However, they can test the low energy SUSY through the precision measurements of Higgs couplings.

The structure of this work is organized as follows. In section 2, we will recapitulate the studies of muon $g-2$ and neutralino DM in the MSSM. In section 3, we perform a numerical scan over the parameter space of EWSUSY and discuss the implications for sparticles. In section 4 , we investigate the prospects of hunting for the electroweakinos and sleptons in those scenarios at the LHC and future colliders. Finally, we draw our conclusions in section 5 .

\section{2 neutralino dark matter and muon $g-2$ in the MSSM}

In the MSSM there are four neutralinos $\tilde{\chi}_{1,2,3,4}^{0}$ that are the mixtures of bino $(\tilde{B})$, wino $\left(\tilde{W}^{0}\right)$ and neutral higgsinos $\left(\tilde{H}_{u, d}^{0}\right)$. The mass matrix is given by [37]

$$
M_{\tilde{\chi}^{0}}=\left(\begin{array}{cccc}
M_{1} & 0 & -c_{\beta} s_{W} m_{Z} & s_{\beta} s_{W} m_{Z} \\
0 & M_{2} & c_{\beta} c_{W} m_{Z} & -s_{\beta} c_{W} m_{Z} \\
-c_{\beta} s_{W} m_{Z} & c_{\beta} c_{W} m_{Z} & 0 & -\mu \\
s_{\beta} s_{W} m_{Z} & -s_{\beta} c_{W} m_{Z} & -\mu & 0
\end{array}\right)
$$

where $s_{\beta}, c_{\beta}, s_{W}$ and $c_{W}$ stands respectively for $\sin \beta, \cos \beta, \sin \theta_{W}$ and $\cos \theta_{W} \cdot M_{1}$ and $M_{2}$ are the soft-breaking mass parameters for bino and wino, respectively. $\mu$ is the higgsino mass parameter. We can diagonalize eq. (2.1) by a unitary $4 \times 4$ matrix $N$. Besides, the mass matrix of charginos that are the mixtures of wino $\left(\tilde{W}^{ \pm}\right)$and charged higgsinos $\left(\tilde{H}_{d}^{-}\right.$, $\tilde{H}_{u}^{+}$) can be written as

$$
M_{\tilde{\chi}^{ \pm}}=\left(\begin{array}{cc}
M_{2} & \sqrt{2} s_{\beta} m_{W} \\
\sqrt{2} c_{\beta} m_{W} & \mu
\end{array}\right)
$$

which can be diagonalized by two unitary $2 \times 2$ matrices $U$ and $V$. 
In the MSSM the lightest sparticle (LSP) can be the neutralino $\tilde{\chi}_{1}^{0}$, which can play the role of dark matter. It is a mixture of bino, wino and higgsinos. Depending on its dominant component, the LSP $\tilde{\chi}_{1}^{0}$ can be bino-like, higgsino-like or wino-like. When $\tilde{\chi}_{1}^{0}$ is wino-like or higgsino-like, it usually has too large annihilation rates in the early universe to produce sufficient dark matter relic density. If we require them to provide the correct dark matter abundance without other non-SUSY dark matter components like axions, the masses of higgsino-like and wino-like dark matter have to be at $\mathrm{TeV}$ scale $[38,39]$, which results in too heavy electroweakino spectrum to generate sizable contributions to muon $g-2$. On the other hand, the wino-like or higgsino-like dark matter scattering with nucleon has a sizable cross section and thus subject to stringent limits from dark matter direct detection experiments. Besides, it should be noted that the sneutrino in our study can be dark matter as well, which, however, was excluded by the direct detection. Therefore, in our study, we will focus on the bino-like dark matter, which can give the observed relic density by mixing with higgsino/wino, resonantly annihilating through $Z$ /Higgs bosons or coannihilating with other light sparticles. The first two mechanisms have been tightly constrained by current XENON1T and LHC experiments [40-42], while the coannihilation with light sparticles can still be consistent with current data [43].

The SUSY contributions to the muon $g-2$ mainly come from the neutralino-smuon and chargino-sneutrino loops. The expressions of one-loop corrections to $a_{\mu}$ are given by [5]

$$
\begin{aligned}
\delta a_{\mu}^{\tilde{\chi}^{0}} & =\frac{m_{\mu}}{16 \pi^{2}} \sum_{i, m}\left\{-\frac{m_{\mu}}{12 m_{\tilde{\mu}_{m}}^{2}}\left(\left|n_{i m}^{L}\right|^{2}+\left|n_{i m}^{R}\right|^{2}\right) F_{1}^{N}\left(x_{i m}\right)+\frac{m_{\tilde{\chi}_{i}^{0}}^{0}}{3 m_{\tilde{\mu}_{m}}^{2}} \operatorname{Re}\left[n_{i m}^{L} n_{i m}^{R}\right] F_{2}^{N}\left(x_{i m}\right)\right\}, \\
\delta a_{\mu}^{\tilde{\chi}^{ \pm}} & =\frac{m_{\mu}}{16 \pi^{2}} \sum_{k}\left\{\frac{m_{\mu}}{12 m_{\tilde{\nu}_{\mu}}^{2}}\left(\left|c_{k}^{L}\right|^{2}+\left|c_{k}^{R}\right|^{2}\right) F_{1}^{C}\left(x_{k}\right)+\frac{2 m_{\tilde{\chi}_{k}^{ \pm}}^{ \pm}}{3 m_{\tilde{\nu}_{\mu}}^{2}} \operatorname{Re}\left[c_{k}^{L} c_{k}^{R}\right] F_{2}^{C}\left(x_{k}\right)\right\},
\end{aligned}
$$

where

$$
\begin{aligned}
n_{i m}^{R} & =\sqrt{2} g_{1} N_{i 1} X_{m 2}+y_{\mu} N_{i 3} X_{m 1}, \\
n_{i m}^{L} & =\frac{1}{\sqrt{2}}\left(g_{2} N_{i 2}+g_{1} N_{i 1}\right) X_{m 1}^{*}-y_{\mu} N_{i 3} X_{m 2}^{*}, \\
c_{k}^{R} & =y_{\mu} U_{k 2}, \quad c_{k}^{L}=-g_{2} V_{k 1},
\end{aligned}
$$

with $i, m$ and $k$ being the indices respectively for the neutralinos, smuons and charginos mass eigenstates. $y_{\mu}=g_{2} m_{\mu} / \sqrt{2} m_{W} \cos \beta$ being the muon Yukawa coupling. The loop functions $F_{1,2}^{N}$ and $F_{1,2}^{C}$, depending on the variables $x_{i m}=m_{\chi_{i}^{0}}^{2} / m_{\tilde{\mu}_{m}}^{2}, x_{k}=m_{\chi_{k}^{ \pm}}^{2} / m_{\tilde{\nu}_{\mu}}^{2}$, are normalized so that $F_{1,2}^{N}(1)=F_{1,2}^{C}(1)=1$, which can be found in [5]. The unitary matrix $X$ that diagonalizes the smuon mass matrix $M_{\tilde{\mu}}^{2}$ is given by,

$$
X M_{\tilde{\mu}}^{2} X^{\dagger}=\operatorname{diag}\left(m_{\tilde{\mu}_{1}}^{2}, m_{\tilde{\mu}_{2}}^{2}\right),
$$

where

$$
M_{\tilde{\mu}}^{2}=\left(\begin{array}{cc}
m_{L}^{2}+\left(s_{W}^{2}-\frac{1}{2}\right) m_{Z}^{2} \cos 2 \beta & m_{\mu}\left(A_{\mu}^{*}-\mu \tan \beta\right) \\
m_{\mu}\left(A_{\mu}-\mu^{*} \tan \beta\right) & m_{R}^{2}-s_{W}^{2} m_{Z}^{2} \cos 2 \beta
\end{array}\right),
$$


in the $\left\{\tilde{\mu}_{L}, \tilde{\mu}_{R}\right\}$ basis. Assuming all sparticles have an universal mass $M_{\mathrm{SUSY}}$, the SUSY contributions to muon $g-2$ can be approximated as [4]

$$
\delta a_{\mu}^{\mathrm{SUSY}}=\frac{\tan \beta}{192 \pi^{2}} \frac{m_{\mu}^{2}}{M_{\mathrm{SUSY}}^{2}}\left(5 g_{2}^{2}+g_{1}^{2}\right)=14 \tan \beta\left(\frac{100 \mathrm{GeV}}{M_{\mathrm{SUSY}}}\right)^{2} 10^{-10} .
$$

It can be seen that the SUSY contributions can be enhanced by a large $\tan \beta$ and suppressed by SUSY mass scale so that heavy SUSY will decouple from such a low energy observable. To generate sizable contributions to the muon $g-2$, the involved charginos and neutralinos as well as the sleptons cannot be too heavy. From eq. (2.4), we can find that the contribution of chargino-sneutrino loop usually dominates over that of neutralino-slepton loop. But it should be mentioned that a sizable contribution to $g-2$ anomaly can also be from the bino-smuon loop because of the large smuon left-right mixing induced by large $\mu$ [44].

Two-loop corrections to the muon $g-2$ from fermion/sfermion loops in the MSSM are calculated in $[45,46]$. These corrections are also significant and even logarithmically enhanced for heavy sfermions. For different masses of sparticles running in the loops, a few percent correction for squark masses in the few $\mathrm{TeV}$ region can be obtained. Such a non-decoupling behavior is because that the gaugino and higgs couplings can differ from the corresponding gauge and Yukawa couplings when heavy sfermions are integrated out.

\section{Parameter scan for muon $g-2$ and dark matter}

In conjuncture with the requirements of the dark matter relic density and LHC data, we perform our study in the EWSUSY framework, where only electroweakinos and sleptons are light and colored sparticles are heavy. Such a framework allows us to remain agnostic of the detailed UV-physics, yet still capture the features of models for muon $g-2$ and dark matter in the MSSM. We will focus on two promising scenarios: one has bino, winos and sleptons (BWL), and the other has bino, higgsinos and sleptons (BHL). This will narrow down the parameter space of the MSSM and provide a guidance of hunting for electroweakinos and sleptons at the LHC and future colliders. The relevant parameters are scanned in the following ranges:

$$
\begin{aligned}
\text { BWL : } & 0 \mathrm{TeV} \leq M_{1}, \quad M_{2} \leq 3 \mathrm{TeV}, \quad 3 \mathrm{TeV} \leq \mu \leq 5 \mathrm{TeV} \\
\text { BHL : } & 0 \mathrm{TeV} \leq M_{1}, \quad \mu \leq 3 \mathrm{TeV}, \quad 3 \mathrm{TeV} \leq M_{2} \leq 5 \mathrm{TeV}
\end{aligned}
$$

Other SUSY parameters in both scenarios are taken as

$$
\begin{array}{rlrl}
100 \mathrm{GeV} & \leq M_{L_{1,2}}=M_{E_{1,2}} \leq 3 \mathrm{TeV} & 1 & \leq \tan \beta \leq 50 \\
3 \mathrm{TeV} & \leq M_{\widetilde{t}_{R}} \leq 5 \mathrm{TeV} & -5 \mathrm{TeV} & \leq A_{t}=A_{b}=A_{\tau} \leq 5 \mathrm{TeV} \\
M_{L_{3}} & =M_{E_{3}}=M_{3}=5 \mathrm{TeV} & A_{u} & =A_{d}=A_{e}=0
\end{array}
$$

In our scan we consider the following experimental constraints:

(1) We use SUSY-HIT [47] to calculate the mass spectrum and branching ratios of the particles. We require the Higgs boson $h$ to be SM-like and in the range of $122<$ $m_{H}<128 \mathrm{GeV}$. 
(2) We impose the constraint of meta-stability of the vacuum state by demanding $\left|A_{t}\right| \lesssim$ $2.67 \sqrt{M_{\widetilde{Q}_{3 L}}^{2}+M_{\widetilde{t}_{R}}^{2}+M_{A}^{2} \cos ^{2} \beta}[48]$.

(3) The sleptons must be above $100 \mathrm{GeV}$, as required by the LEP2 constraints.

(4) We calculate the dark matter relic density by MicrOMEGAs 4.3.2 [49] and require its value within $2 \sigma$ range of the Planck observed value, $\Omega_{\mathrm{DM}} h^{2}=0.1186 \pm 0.002$ [50].

(5) We require the SUSY contribution to explain the current value of muon $g-2$ data $\delta a_{\mu}^{\exp -\mathrm{SM}}=(2.68 \pm 0.63 \pm 0.43) \times 10^{-9}[2]$ within the $2 \sigma$ range.

In figure 1, we present the contributions of sparticles to the muon $g-2$ for samples survived the constraints (1)-(4) on the plane of $\delta a_{\mu}^{\mathrm{SUSY}}$ versus $m_{\tilde{\chi}_{1}^{0}}$ and $m_{\tilde{\ell}}$. The red shaded areas are the $2 \sigma$ ranges of explaining current muon $g-2$ anomaly. We find that the dark matter abundance in BWL scenario is achieved mainly through the co-annihilation of the bino-like $\tilde{\chi}_{1}^{0}$ and wino-like $\tilde{\chi}_{2}^{0}$ and $\tilde{\chi}_{1}^{ \pm}$. The slepton coannihilation also contributes to the relic density in the relatively heavy mass range. While in the BHL scenario, the correct dark matter abundance is obtained through the co-annihilation of the LSP with sleptons.

In order to interpret the muon $g-2$ deviation, we can see that the masses of $\tilde{\chi}_{1}^{0}$ and $\tilde{\ell}$ have to be lighter than about $700 \mathrm{GeV}$ and $800 \mathrm{GeV}$ in BWL scenario, respectively. But in the BHL scenario, the masses of $\tilde{\chi}_{1}^{0}$ and $\tilde{\ell}$ have to be less than about $350 \mathrm{GeV}$. On the other hand, we find that the smuon with a mass less than about $200 \mathrm{GeV}$ have been excluded in BWL scenario because of the over-enhancement of $g-2$. On the other hand, a lighter smuon can exist in BHL scenario. This is because that the right-handed smuon in BHL scenario will lead to a negative contribution to $g-2$ so that a lighter smuon is needed to compensate for such a suppression.

By assuming the expected central value same as the current result of $g-2$, we also show the projected $2 \sigma$ sensitivity of the E989 experiment at Fermilab that are the regions between the dotted lines. It will further constrain the viable mass ranges of sparticles. To be specific, $\tilde{\chi}_{1}^{0}$ and $\tilde{\ell}$ have to be lighter than about $500 \mathrm{GeV}$ and $600 \mathrm{GeV}$ in BWL scenario, respectively, while in the BHL scenario, the masses of $\tilde{\chi}_{1}^{0}$ and $\tilde{\ell}$ have to be less than about $200 \mathrm{GeV}$. If these turn out to be true, several popular high scale SUSY models, such as the CMSSM, mSUGRA, GMSB and AMSB, have to be extended because their sfermion spectrum that needs to explain the $125 \mathrm{GeV}$ Higgs mass is too heavy to accommodate muon $g-2$.

In figure 2, we plot the spin-independent and spin-dependent LSP-nucleon scattering cross sections of the samples survived the constraints (1)-(5). Since in BWL scenario the higgsinos are rather heavy and the LSP $\tilde{\chi}_{1}^{0}$ is extremely bino-like, it scatters with nucleon very weakly and thus the SI and SD LSP-nucleon scattering cross sections are very small, which can be much below the LZ-projected sensitivities. Those samples may be probed at colliders $[51,52]$. On the other hand, the LSP $\tilde{\chi}_{1}^{0}$ in BHW scenario can have certain higgsino component so that it can scatter with the nucleons sizably and are tightly constrained by current direct detection limits. 

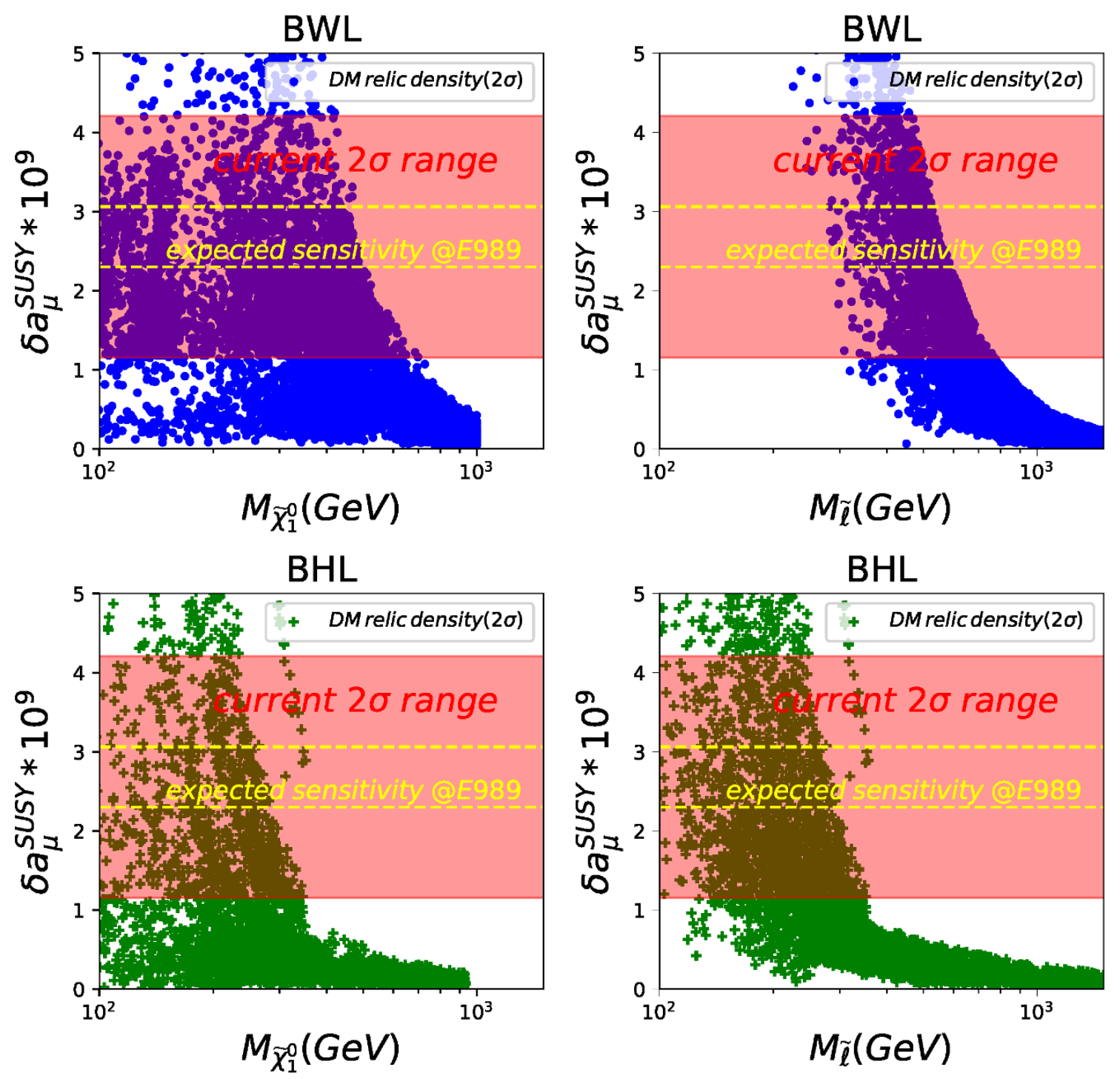

Figure 1. Scatter plots of the two types of samples survived the constraints (1)-(4) on the plane of $\delta a_{\mu}^{\text {SUSY }}$ versus $m_{\tilde{\chi}_{1}^{0}}$ and $m_{\tilde{\ell}}$, showing the contributions to the muon $g-2$. The shaded areas are the current $2 \sigma$ ranges, while the regions between the dotted lines are the projected $2 \sigma$ sensitivity of the experiment at Fermilab (E989), where the expected central value is assumed same as the current experimental value.

\section{4 observabilities at LHC upgrades and Higgs factory}

In figure 3, we display the samples survived the constraints (1)-(5) and the dark matter direct detection. The BWL scenario is shown in the upper panel of figure 3 , where $\tilde{\chi}_{1}^{0}$ is bino-like and $\tilde{\chi}_{2}^{0}$ is wino-like. For most samples the mass difference between the winolike $\tilde{\chi}_{2}^{0}$ and the bino-like $\tilde{\chi}_{1}^{0}$ is rather small, while the smuon mass can be quite near or significantly heavier than the mass of $\tilde{\chi}_{1}^{0}$. The BHL scenario is shown in the lower panel of figure 3, which has a light spectrum of bino and sleptons but with heavy higgsinos. In this scenario $\tilde{\chi}_{1}^{0}$ is also rather bino-like, albeit with small higgsino admixture, while the $\tilde{\chi}_{2}^{0}$ is higgsino-like. The mass difference between the LSP and sleptons is quite small as well. 

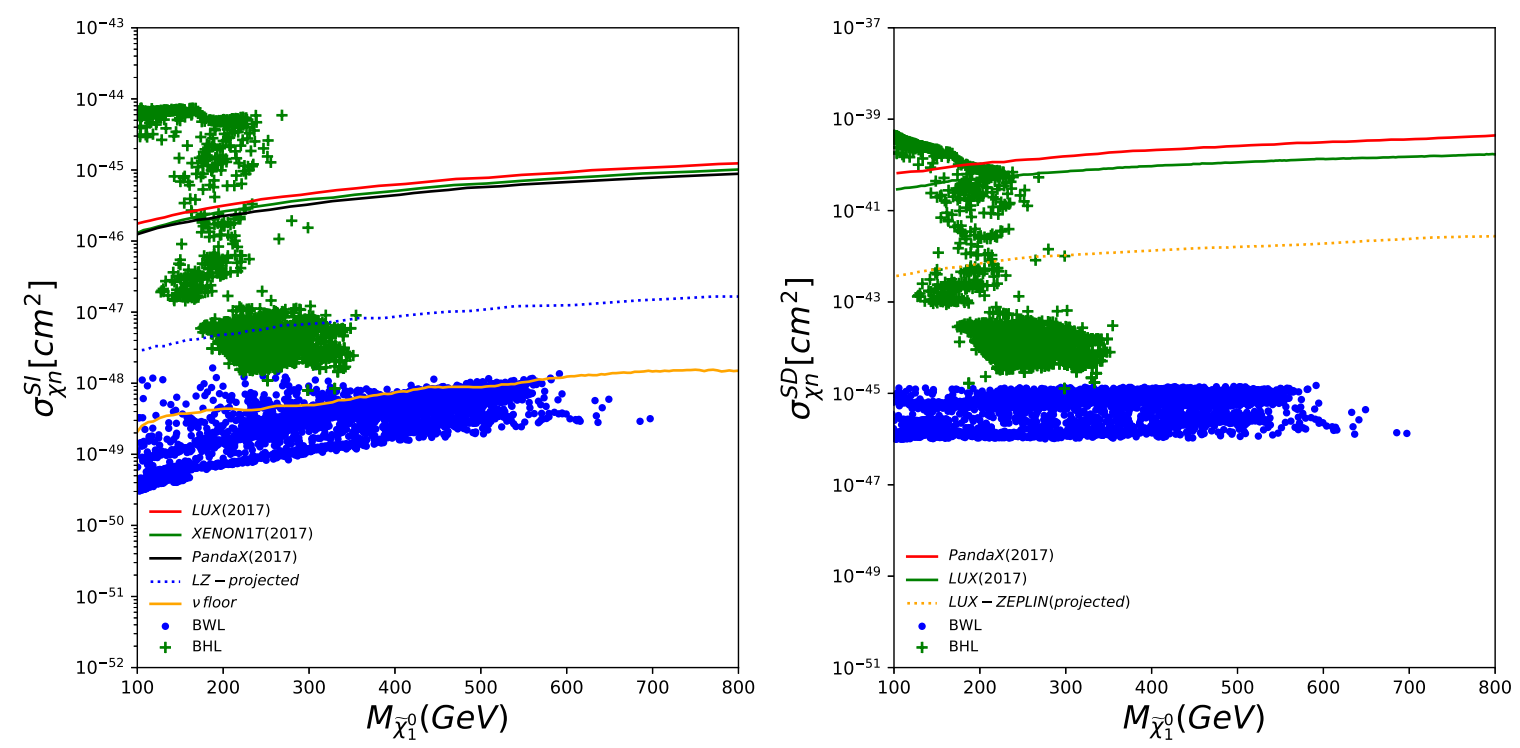

Figure 2. Scatter plots of the samples survived the constraints (1)-(5), showing the spinindependent and spin-dependent neutralino LSP-nucleon scattering cross sections. The samples in BWL and BHL scenarios are denoted by blue dots and green plus respectively. The observed $90 \%$ CL upper limits from LUX2017 [53], XENON1T-2017 [54] and PandaX-2017 (Run9+Run10) [55] and the future sensitivities from LZ-projected [56] are shown.

We also present the latest exclusion limits from the null results of searching for slepton pair and wino pair at $13 \mathrm{TeV}$ LHC with the luminosity of $139 \mathrm{fb}^{-1}$. For the BWL scenario, a large portion of samples with sizable mass splitting of slepton and LSP have been excluded, which implies a compressed spectrum of bino, wino and sleptons. In the meanwhile, the light wino-like $\tilde{\chi}_{2}^{0}$ in the coannihilation region are not allowed either. On the other hand, there is no constraint on the BHL scenario at the LHC because the samples have either heavy higgsino-like $\tilde{\chi}_{2}^{0}$ or compressed slepton and LSP with masses being larger than about $200 \mathrm{GeV}$.

Next, we investigate the observability of the BWL and BHL scenarios at $27 \mathrm{TeV}$ HELHC with $15 \mathrm{ab}^{-1}$ and Higgs factory. Given the production cross section of the wino pair are larger than that of slepton pair, we perform a detailed Monte Carlo simulation of the process $p p \rightarrow j \tilde{\chi}_{2}^{0}\left(\rightarrow Z^{*} \tilde{\chi}_{1}^{0} \rightarrow \ell^{+} \ell^{-} \tilde{\chi}_{1}^{0}\right) \tilde{\chi}_{1}^{ \pm}\left(\rightarrow W^{*} \tilde{\chi}_{1}^{0} \rightarrow q \bar{q}+\tilde{\chi}_{1}^{0}\right) \rightarrow j+\ell^{+} \ell^{-}+\mathscr{E}_{T}$ for the compressed bino-wino in BWL scenario. While since the sleptons are much lighter than the higgsinos in the BHL, we will analyze the process $p p \rightarrow j \tilde{\ell}\left(\rightarrow \ell^{-} \tilde{\chi}_{1}^{0}\right) \tilde{\ell}^{*}\left(\rightarrow \ell^{+} \tilde{\chi}_{1}^{0}\right) \rightarrow j+\ell^{+} \ell^{-}+\mathbb{E}_{T}$ for the compressed bino-slepton in the BHL scenario. The schematic diagram of those two process are shown in figure 4 . So in both signal processes, there are a pair of soft oppositesign same-flavor leptons plus jets plus large missing transverse energy. We will utilize these features to enhance the sensitivity of our signals. The main SM backgrounds come from the Drell-Yan processes, dibosons and the leptonic $t \bar{t}$ events. We generate parton-level events by using MadGraph5_aMC@NLO [59] and then the events are passed to Pythia [60] for showering and hadronization. The detector effects are simulated by Delphes [61]. We perform the analysis of events in the framework of CheckMATE2 [62-64], and evaluate the 

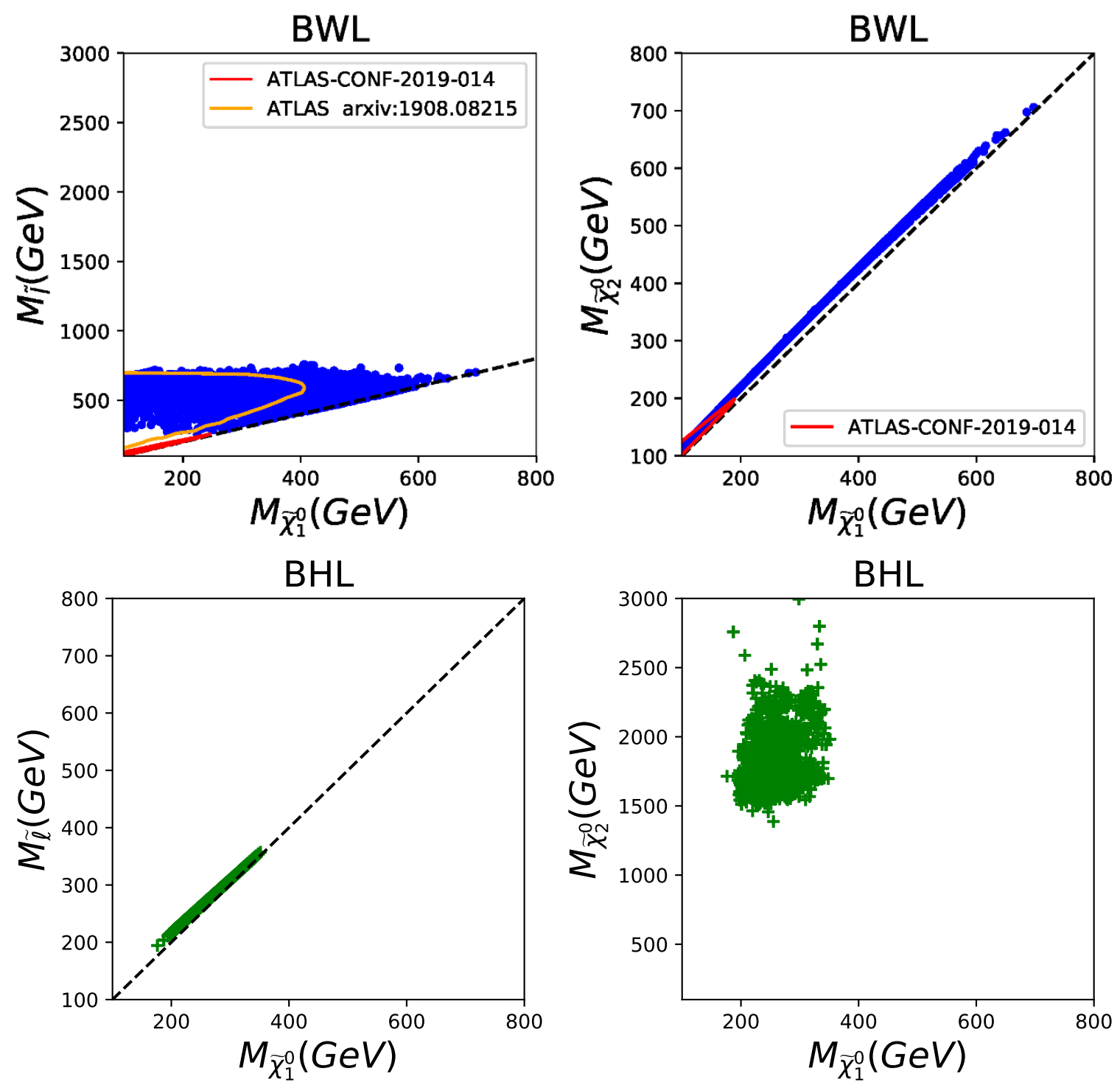

Figure 3. Scatter plots of the samples survived the constraints (1)-(5) and the current direct detection limits, displayed on the plane of $M_{\tilde{\chi}_{1}^{0}}$ versus $M_{\tilde{\chi}_{2}^{0}}$ and $M_{\tilde{\ell}}$. The upper and lower panels correspond to the BWL and BHL scenarios, respectively. For the BWL case the regions excluded by ATLAS [57, 58] are shown.

significance by

$$
Z=\frac{S}{\sqrt{S+B+(\beta B)^{2}}},
$$

where $\beta$ stands for the expected systematic uncertainty. It has to be revisited with the real performance of the upgraded LHC detectors. As a theoretical estimation, we take $\beta=10 \%$ in our calculations.

In figure 5, we show the normalized distributions of the missing transverse energy $\mathbb{E}_{T}$ and the dilepton invariant mass $m_{\ell \ell}$ of the signal and background events. We find that both signals have more events in the range of the large $\mathbb{E}_{T}$, which can highly suppress the 

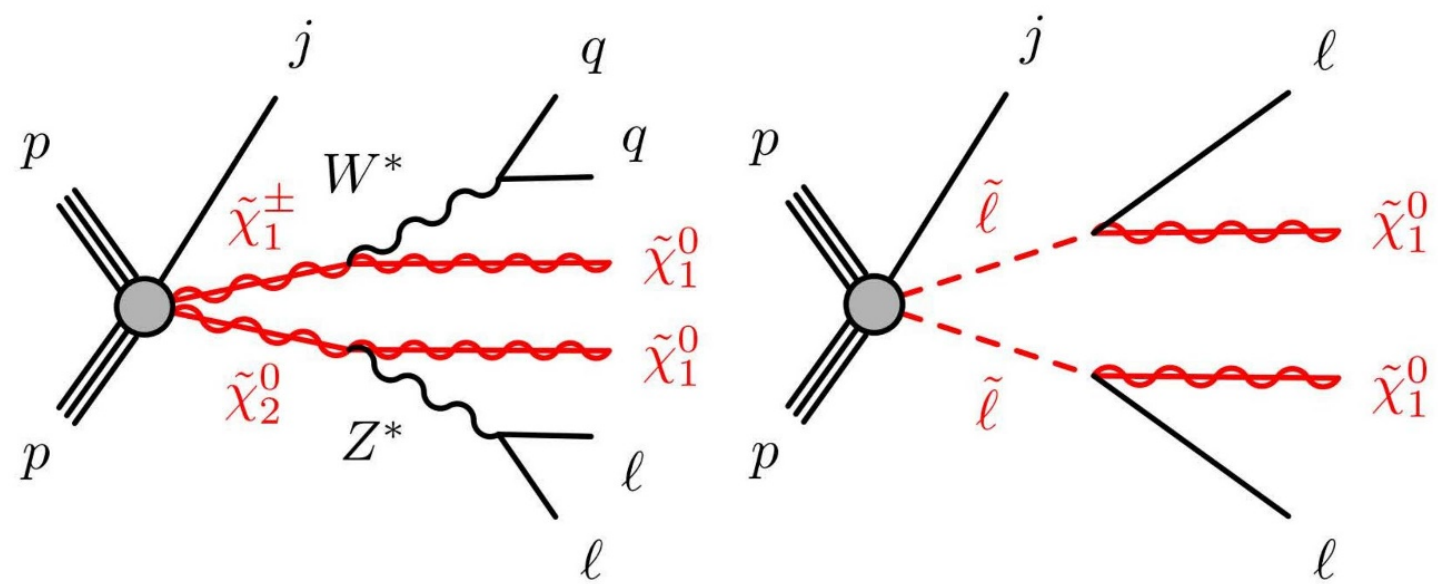

Figure 4. The schematic diagrams of the wino pair production process $p p \rightarrow j \tilde{\chi}_{2}^{0} \tilde{\chi}_{1}^{ \pm}$in the BWL scenario (left panel) and the slepton pair production process $p p \rightarrow j \tilde{\ell}^{*}\left(\tilde{\ell}=\tilde{e}_{1}, \tilde{\mu}_{1}\right)$ in the BHL scenario (right panel).
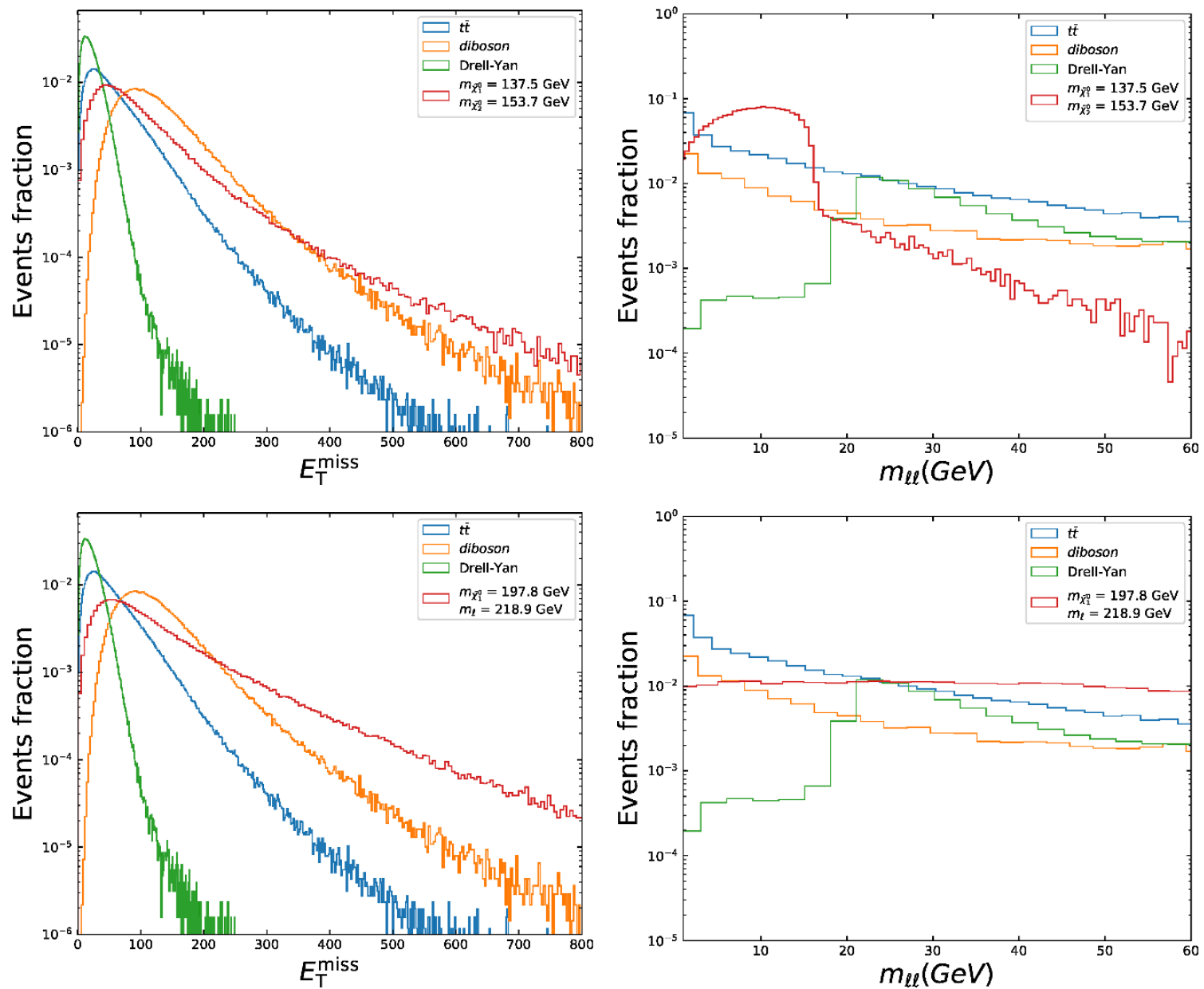

Figure 5. The normalized distributions of $\mathbb{E}_{T}$ and $m_{\ell \ell}$ of the signal and background events at the $27 \mathrm{TeV}$ HE-LHC. The upper and lower panels are for the BWL and BHL scenarios, respectively. 


\begin{tabular}{|lrrrr|}
\hline Cuts & $t \bar{t}$ & diboson & Drell-Yan & BWL \\
\hline $\mathbb{E}_{T}>200 \mathrm{GeV}$ & 37512.99 & 1721.53 & 246.54 & 618.83 \\
\hline$N(\ell)=2$, OSSF, $p_{T}\left(\ell_{1}\right)>5 \mathrm{GeV}, p_{T}\left(\ell_{2}\right)>4 \mathrm{GeV}$ & 956.16 & 38.536 & 15.60 & 51.03 \\
\hline $\begin{array}{l}N(j) \geq 1, N(b)=0, p_{T}\left(j_{1}\right)>100 \mathrm{GeV}, \\
\Delta \phi\left(j_{1}, P_{T}^{\text {miss }}\right)>2, \Delta \phi\left(j, P_{T}^{\text {miss }}\right)>0.4\end{array}$ & & & & \\
$m_{\tau \tau} \notin[0,160) \mathrm{GeV}, 1 \mathrm{GeV}<m_{\ell \ell}<60 \mathrm{GeV}$, & & & & \\
$m_{\ell \ell} \notin(3,3.2) \mathrm{GeV}, \Delta R_{\ell \ell}>0.05$ & 23.84 & 3.64 & 3.12 & 27.68 \\
\hline $\mathbb{E}_{T} / H_{T}^{\text {lep }}>\max \left(5,15-2 m_{\ell \ell}\right)$ & 7.94 & 2.26 & 3.12 & 24.22 \\
\hline$\Delta R_{\ell \ell}<2$ & 2.65 & 1.38 & 3.12 & 20.15 \\
\hline
\end{tabular}

Table 1. The cut flow for the cross sections of the signal and backgrounds at the $27 \mathrm{TeV}$ HE-LHC for the BWL benchmark point $m_{\tilde{\chi}_{1}^{0}}=137.4 \mathrm{GeV}, m_{\tilde{\chi}_{2}^{0}}=m_{\tilde{\chi}_{1}^{ \pm}}=153.7 \mathrm{GeV}, \tan \beta=50$. The cross sections are in units of $\mathrm{fb}$.

Drell-Yan and $t \bar{t}$ backgrounds. In additional, due to two soft leptons decaying from the sleptons, both signals predict a small value of $m_{\ell \ell}$. According to the kinematical features, we impose the following event selection criteria:

- We require the missing transverse energy $\mathbb{E}_{T}>200 \mathrm{GeV}$.

- Two opposite-sign same-flavor (OSSF) leptons are required. The leading and subleading leptons should have the transverse momentum $p_{T}\left(\ell_{1}\right)>5 \mathrm{GeV}$ and $p_{T}\left(\ell_{2}\right)>$ $4 \mathrm{GeV}$. The angular distance $0.05<\Delta R\left(\ell_{1}, \ell_{2}\right)<2$ in BWL scenario and $0.05<$ $\Delta R\left(\ell_{1}, \ell_{2}\right)$ in BHL scenario are required.

- We require at least one jet and the leading jet $p_{T}\left(j_{1}\right)>100 \mathrm{GeV}$. The angular separations have to be $\Delta \phi\left(j_{1}, P_{T}^{\text {miss }}\right)>2$ and $\Delta \phi\left(j, P_{T}^{\text {miss }}\right)>0.4$. Also we veto $b$-jets to reduce $t \bar{t}$ background.

- We require the dilepton invariant mass $1 \mathrm{GeV}<m_{\ell \ell}<60 \mathrm{GeV}$ and $m_{\ell \ell} \notin(3,3.2) \mathrm{GeV}$ to suppress contributions from $J / \psi$ decays and on-shell $Z$ boson decays.

- The scalar sum of the lepton transverse momenta $H_{T}^{\text {lep }}=p_{T}^{\ell_{1}}+p_{T}^{\ell_{2}}$ is small in the compressed region. The ratio $E_{T}^{\text {miss }} / H_{T}^{\text {lep }}$ can improve the sensitivity for smaller mass splitting. We require $\mathbb{E}_{T} / H_{T}^{\text {lep }}>\max \left[5,15-2 m_{\ell \ell} /(1 \mathrm{GeV})\right]$ for wino pair and $\mathbb{E}_{T} / H_{T}^{\text {lep }}>\max \left[3,15-2\left[m_{T_{2}}^{100} /(1 \mathrm{GeV})-100\right]\right]$ for slepton pair, where the stransverse mass is defined in [65-67].

- The invariant mass $m_{\tau \tau} \notin[0,160) \mathrm{GeV}$ can suppress the Drell-Yan background.

In tables 1 and 2, we demonstrate the cut flows for the benchmark points in two scenarios. We can see that the soft OSSF leptons cut will significantly reduce all backgrounds, in particular for $t \bar{t}$ events. The hard $p_{T}\left(j_{1}\right)>100 \mathrm{GeV}$ and small dilepton invariant mass $1 \mathrm{GeV}<m_{\ell \ell}<60 \mathrm{GeV}$ can further suppress $t \bar{t}$ and diboson backgrounds by about one order. As pointed in $[57,58]$, the observable $\mathbb{E}_{T} / H_{T}^{\text {lep }}$ is useful for reducing the $t \bar{t}$ background. 


\begin{tabular}{|c|c|c|c|c|}
\hline Cuts & $t \bar{t}$ & diboson & Drell-Yan & BHL \\
\hline $\mathbb{E}_{T}>200 \mathrm{GeV}$ & 37512.99 & 1721.53 & 246.54 & 69.97 \\
\hline$N(\ell)=2, \mathrm{OSSF}, p_{T}\left(\ell_{1}\right)>5 \mathrm{GeV}, p_{T}\left(\ell_{2}\right)>4 \mathrm{GeV}$ & 956.16 & 38.54 & 15.60 & 7.21 \\
\hline$N(j) \geq 1, N(b)=0, p_{T}\left(j_{1}\right)>100 \mathrm{GeV}$ & & & & \\
\hline$\Delta \phi\left(j_{1}, P_{T}^{\mathrm{miss}}\right)>2, \Delta \phi\left(j, P_{T}^{\mathrm{miss}}\right)>0.4$ & 74.16 & 16.43 & 9.36 & 4.71 \\
\hline $\begin{array}{l}m_{\tau \tau} \notin[0,160) \mathrm{GeV}, 1 \mathrm{GeV}<m_{\ell \ell}<60 \mathrm{GeV} \\
m_{\ell \ell} \notin(3,3.2) \mathrm{GeV}, \Delta R_{\ell \ell}>0.05\end{array}$ & 23.84 & 3.64 & 3.12 & 2.18 \\
\hline $\mathbb{E}_{T} / H_{T}^{\mathrm{lep}}>\max \left[3,15-2\left[m_{T_{2}}^{100} /(1 \mathrm{GeV})-100\right]\right]$ & 13.24 & 3.15 & 3.12 & 1.73 \\
\hline
\end{tabular}

Table 2. The cut flow for the cross sections of the signal and backgrounds at the $27 \mathrm{TeV}$ HE-LHC for the BHL benchmark point $m_{\tilde{\chi}_{1}^{0}}=197.8 \mathrm{GeV}, m_{\tilde{\ell}}=218.9 \mathrm{GeV}, \tan \beta=17.5$. The cross sections are in units of $\mathrm{fb}$.

In figure 6 , we display the significances of the processes $p p \rightarrow j \tilde{\chi}_{2}^{0} \tilde{\chi}_{1}^{ \pm}$and $p p \rightarrow j \tilde{\ell} \tilde{\ell}^{*}$ at the HL-LHC and HE-LHC. It can be seen that a portion of the samples in both scenarios will be excluded by the search for soft lepton pair plus missing energy events at the HLLHC. The future HE-LHC is able to further exclude the whole parameter space of BHL and most part of BWL scenarios for satisfying muon $g-2$ and DM experimental results within $2 \sigma$ level. We also checked that the $100 \mathrm{TeV}$ proton-proton collider SPPC with the same luminosty cannot do much better than the HE-LHC due to the enhanced backgrounds. On the other hand, it should be mentioned that the heavy higgsinos decaying to light bino in the BHL scenario will provide $3 \ell+\mathbb{E}_{T}$ signature at a $100 \mathrm{TeV}$ hadron collider, which can exclude the higgsino mass up to about $3 \mathrm{TeV}$ at $95 \%$ C.L. . Besides conventional cutbased analysis, the machine learning methods have been recently proposed to enhance the sensitivity in the search of sparticles at the LHC [68-72]. We expect that our result may be improved by using those advanced analysis approaches.

Since the LHC experiment has been continuously pushing up the new physics scale, the future $e^{+} e^{-}$Higgs factory, either CEPC, FCC-ee or ILC, has limited energy to directly produce new particles. However, due to its clean environment, such a Higgs factory is a precision test machine and can measure the Higgs couplings at one percent level or better, which may reveal the new physics effects through the Higgs couplings (see examples, [77-82]).

As shown in the above section, the electroweakinos and sleptons cannot be too heavy in order to explain the muon $g-2$ and provide the correct dark matter abundance. These light uncolored SUSY particles may cause some indirect effects in the Higgs couplings. Among the Higgs couplings, the $h b \bar{b}$ and $h \tau^{+} \tau^{-}$couplings can still deviate from the SM predictions sizably [83]. In the following we demonstrate the $h b \bar{b}$ coupling as an illustration.

In figure 7 , we display the $h b \bar{b}$ coupling for the samples in figure 6 that cannot be excluded at the HE-LHC. At tree level the $h b \bar{b}$ coupling is given by $g\left(m_{b} / 2 m_{W}\right)(\sin \alpha / \cos \beta)$ and the one-loop corrections are presented in [84]. In our calculations we use the package FeynHiggs-2.11.3 [85] which includes the one-loop effects and also various two-loop contributions. The sensitivities of the HL-LHC $\left(14 \mathrm{TeV}, 3 \mathrm{ab}^{-1}\right)$, ILC $\left(250 \mathrm{GeV}, 2 \mathrm{ab}^{-1}\right)$, FCC-ee 
BWL

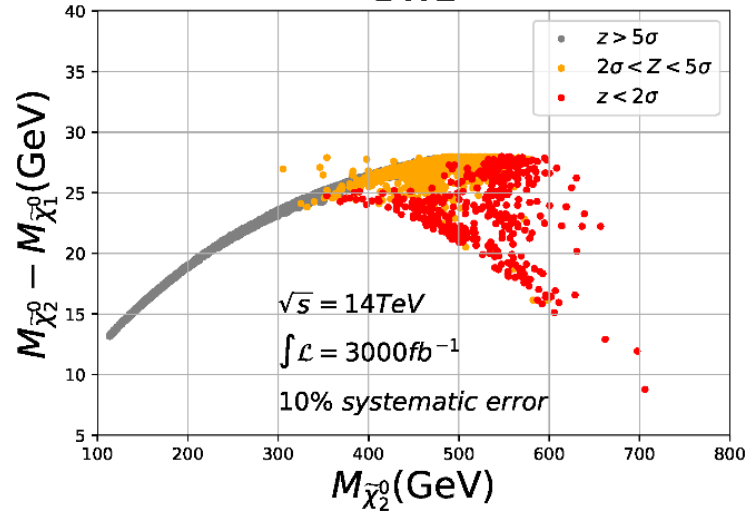

BWL

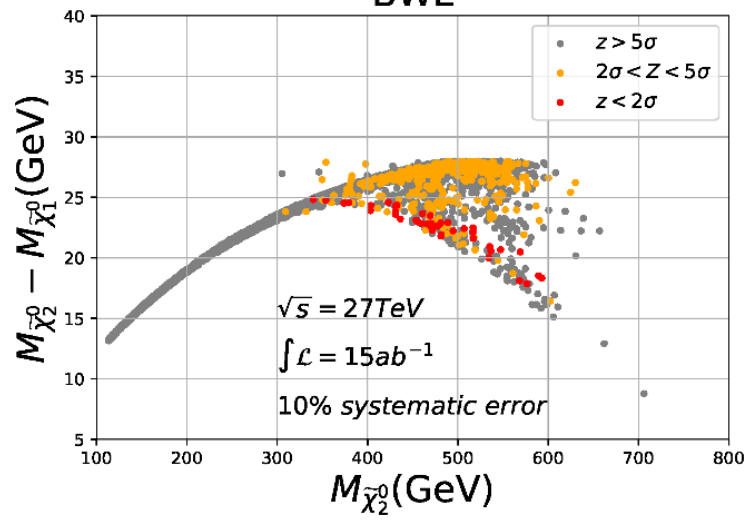

BHL

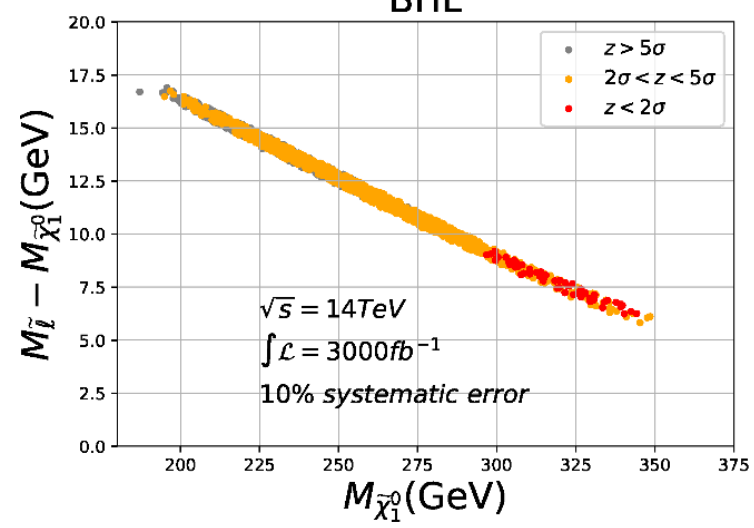

$\mathrm{BHL}$

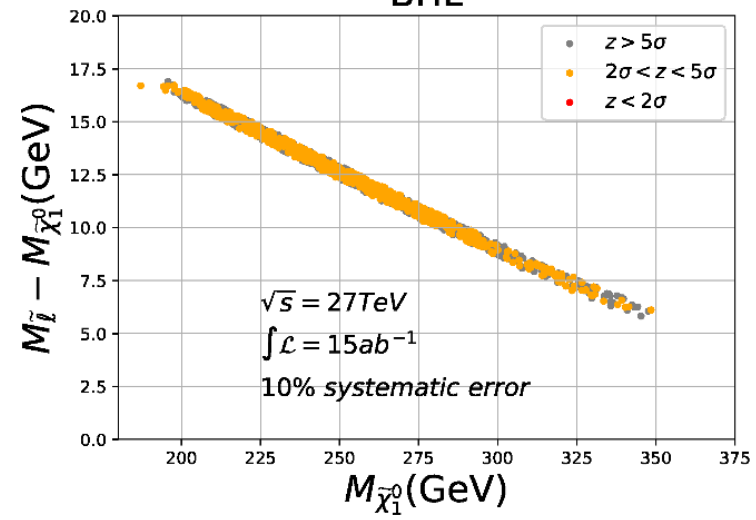

Figure 6. Same as figure 3, but showing the significance of the processes $p p \rightarrow \tilde{\chi}_{2}^{0} \tilde{\chi}_{1}^{ \pm}+$jets and $p p \rightarrow \tilde{\ell} \tilde{\ell}+$ jets at the HL-LHC and HE-LHC. The left and right panels are for the BWL and BHL scenarios, respectively.

$\left(240 \mathrm{GeV}, 5 \mathrm{ab}^{-1}\right)$ and CEPC $\left(240 \mathrm{GeV}, 5 \mathrm{ab}^{-1}\right)$ to the Higgs couplings are also shown. We can see that in the BWL scenario the $h b \bar{b}$ coupling can still be enhanced by about two percent, which is below the HL-LHC sensitivity but can be readily covered by the Higgs factory ILC, FCC-ee, or CEPC. Therefore, the precision measurement of the Higgs couplings could play a complementary role of probing such a scenario at future high energy lepton collider.

\section{Conclusions}

Since the colored sparticles have been excluded up to $\mathrm{TeV}$ scale, searching for the electroweak supersymmetry will be one of the major tasks in future experiments. Besides the LHC, the on-going muon $g-2$ and dark matter experiments provide another good place to hunt for electroweakinos and sleptons in EWSUSY. In this work, we examined the parameter space of EWSUSY under the constraints of the muon $g-2$ anomaly, the DM relic density, the DM direct detections and the LHC data. By analyzing the survived samples, we obtained the following observations: (1) There are two viable scenarios for explaining the muon $g-2$ anomaly. One has the light compressed bino, winos and sleptons 


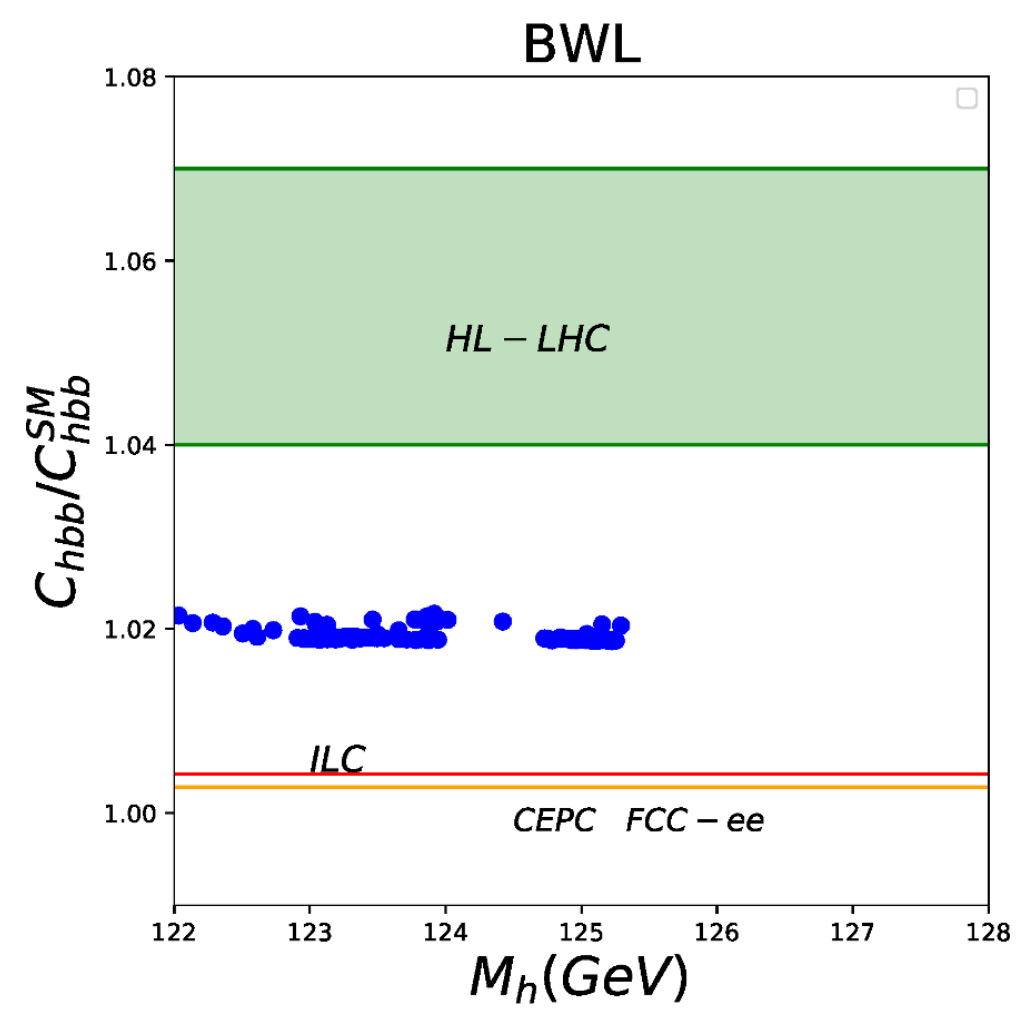

Figure 7. The reduced SM-like Higgs coupling $C_{h b \bar{b}} / C_{h b \bar{b}}^{\mathrm{SM}}$ of the samples with the significance $Z<2 \sigma$ for the BWL scenario in figure 6. The sensitivities of the HL-LHC (14 TeV, $\left.3 \mathrm{ab}^{-1}\right)$ [73], ILC $\left(250 \mathrm{GeV}, 2 \mathrm{ab}^{-1}\right)$ [74], FCC-ee $\left(240 \mathrm{GeV}, 5 \mathrm{ab}^{-1}\right)$ [75] and CEPC $\left(240 \mathrm{GeV}, 5 \mathrm{ab}^{-1}\right)$ [76] to the Higgs couplings are also shown.

(BWL), and the other has light compressed bino and sleptons but heavy higgsinos (BHL). In the BHL scenario, the masses of $\tilde{\chi}_{1}^{0}$ and $\tilde{\ell}$ have to be smaller than about $350 \mathrm{GeV}$. In the BWL scenario, the masses of $\tilde{\chi}_{1}^{0}$ and $\tilde{\ell}$ have to be smaller than about $700 \mathrm{GeV}$ and $800 \mathrm{GeV}$, respectively. If this anomaly persists in the on-going E989 experiment, the allowed parameter space will be further narrowed. (2) In both scenarios, the dark matter has to be the bino-like neutralino and the dominant annihilation mechanism to achieve the correct dark matter abundance is through the bino-wino or bino-slepton coannihilation. Also, we found that the sneutrino DM or the neutralino DM with sizable higgsino component has been excluded by direct detections, due to the large scattering cross section of dark matter and nucleus. (3) The BWL scenario has been tightly constrained by the latest LHC Run-2 results of searches for soft $\ell^{+} \ell^{-}+\mathbb{E}_{T}$ events from slepton pair, which implies a compressed spectrum of bino, winos and sleptons. In contrast, the BHL scenario can escape the current LHC limits. We explored the observability of these sparticles in both scenarios at future colliders. We found that the HE-LHC with the luminosity $L=15 \mathrm{ab}^{-1}$ can exclude the whole BHL scenario and most part of BWL scenarios at $2 \sigma$ level. The rest of samples that alter the Higgs coupling by two percent level may be excluded by the precision measurement of the Higgs couplings at a future Higgs factory. 


\section{Acknowledgments}

We thank Chengcheng Han and Jie Ren for useful discussions. Part of this work was done while M.A. was visiting Nanjing Normal University. This work was supported by the National Natural Science Foundation of China (NNSFC) under grant Nos. 11705093, 11675242, 11821505, and 11851303, by Peng-Huan-Wu Theoretical Physics Innovation Center (11847612), by the CAS Center for Excellence in Particle Physics (CCEPP), by the CAS Key Research Program of Frontier Sciences and by a Key R\&D Program of Ministry of Science and Technology under number 2017YFA0402200-04.

Open Access. This article is distributed under the terms of the Creative Commons Attribution License (CC-BY 4.0), which permits any use, distribution and reproduction in any medium, provided the original author(s) and source are credited.

\section{References}

[1] A. Driutti, Status of the Muon g- 2 experiment at Fermilab, SciPost Phys. Proc. 1 (2019) 033 [FERMILAB-CONF-18-623-E] [INSPIRE].

[2] Particle Data Group collaboration, Review of Particle Physics, Phys. Rev. D 98 (2018) 030001 [INSPIRE].

[3] G. Arcadi et al., The waning of the WIMP? A review of models, searches and constraints, Eur. Phys. J. C 78 (2018) 203 [arXiv:1703.07364] [INSPIRE].

[4] T. Moroi, The Muon anomalous magnetic dipole moment in the minimal supersymmetric standard model, Phys. Rev. D 53 (1996) 6565 [Erratum ibid. D 56 (1997) 4424] [hep-ph/9512396] [INSPIRE].

[5] S.P. Martin and J.D. Wells, Muon Anomalous Magnetic Dipole Moment in Supersymmetric Theories, Phys. Rev. D 64 (2001) 035003 [hep-ph/0103067] [inSPIRE].

[6] D. Stöckinger, The Muon Magnetic Moment and Supersymmetry, J. Phys. G 34 (2007) R45 [hep-ph/0609168] [INSPIRE].

[7] P. Athron et al., GM2Calc: Precise MSSM prediction for $(g-2)$ of the muon, Eur. Phys. J. C 76 (2016) 62 [arXiv: 1510.08071] [InSPIRE].

[8] ATLAS collaboration, Search for squarks and gluinos in final states with jets and missing transverse momentum using $139 \mathrm{fb}^{-1}$ of $\sqrt{\mathrm{s}}=13 \mathrm{TeV}$ pp collision data with the ATLAS detector, ATLAS-CONF-2019-040.

[9] GAMBIT collaboration, Combined collider constraints on neutralinos and charginos, Eur. Phys. J. C 79 (2019) 395 [arXiv: 1809.02097] [InSPIRE].

[10] T.T. Yanagida and N. Yokozaki, Muon g- 2 in MSSM gauge mediation revisited, Phys. Lett. B 772 (2017) 409 [arXiv: 1704.00711] [InSPIRE].

[11] F. Wang, W. Wang and J.M. Yang, Solving the muon $g-2$ anomaly in deflected anomaly mediated SUSY breaking with messenger-matter interactions, Phys. Rev. D 96 (2017) 075025 [arXiv: 1703.10894] [INSPIRE].

[12] F. Wang, W. Wang, J.M. Yang and Y. Zhang, Heavy colored SUSY partners from deflected anomaly mediation, JHEP 07 (2015) 138 [arXiv:1505.02785] [INSPIRE]. 
[13] F. Wang, W. Wang and J.M. Yang, Reconcile muon g-2 anomaly with LHC data in SUGRA with generalized gravity mediation, JHEP 06 (2015) 079 [arXiv: 1504.00505] [INSPIRE].

[14] M. Ibe, T.T. Yanagida and N. Yokozaki, Muon g-2 and $125 \mathrm{GeV}$ Higgs in Split-Family Supersymmetry, JHEP 08 (2013) 067 [arXiv:1303.6995] [INSPIRE].

[15] S. Akula and P. Nath, Gluino-driven radiative breaking, Higgs boson mass, muon $g-2$ and the Higgs diphoton decay in supergravity unification, Phys. Rev. D 87 (2013) 115022 [arXiv: 1304.5526] [INSPIRE].

[16] S. Mohanty, S. Rao and D.P. Roy, Reconciling the muon $g-2$ and dark matter relic density with the LHC results in nonuniversal gaugino mass models, JHEP 09 (2013) 027 [arXiv:1303.5830] [INSPIRE].

[17] K. Kowalska, L. Roszkowski, E.M. Sessolo and A.J. Williams, GUT-inspired SUSY and the muon $g-2$ anomaly: prospects for LHC 14 TeV, JHEP 06 (2015) 020 [arXiv: 1503.08219] [INSPIRE].

[18] F. Wang, L. Wu, J.M. Yang and M. Zhang, $750 \mathrm{GeV}$ diphoton resonance, $125 \mathrm{GeV}$ Higgs and muon $g-2$ anomaly in deflected anomaly mediation SUSY breaking scenarios, Phys. Lett. B 759 (2016) 191 [arXiv: 1512.06715] [InSPIRE].

[19] N. Okada, S. Raza and Q. Shafi, Particle Spectroscopy of Supersymmetric SU(5) in Light of 125 GeV Higgs and Muon g-2 Data, Phys. Rev. D 90 (2014) 015020 [arXiv:1307.0461] [INSPIRE].

[20] I. Gogoladze, F. Nasir, Q. Shafi and C.S. Un, Nonuniversal Gaugino Masses and Muon g-2, Phys. Rev. D 90 (2014) 035008 [arXiv: 1403.2337] [INSPIRE].

[21] K.S. Babu, I. Gogoladze, Q. Shafi and C.S. Ün, Muon g-2, $125 \mathrm{GeV}$ Higgs boson and neutralino dark matter in a flavor symmetry-based MSSM, Phys. Rev. D 90 (2014) 116002 [arXiv: 1406.6965] [INSPIRE].

[22] M.A. Ajaib, I. Gogoladze, Q. Shafi and C.S. Ün, Split sfermion families, Yukawa unification and muon $g-2$, JHEP 05 (2014) 079 [arXiv: 1402 .4918] [INSPIRE].

[23] M. Adeel Ajaib, I. Gogoladze and Q. Shafi, GUT-inspired supersymmetric model for $h \rightarrow \gamma \gamma$ and the muon $g$ - 2, Phys. Rev. D 91 (2015) 095005 [arXiv:1501.04125] [INSPIRE].

[24] I. Gogoladze, Q. Shafi and C.S. Ün, Reconciling the muon $g-2$, a $125 \mathrm{GeV}$ Higgs boson and dark matter in gauge mediation models, Phys. Rev. D 92 (2015) 115014 [arXiv:1509.07906] [INSPIRE].

[25] N. Abe and M. Endo, Recent muon $g-2$ result in deflected anomaly mediated supersymmetry breaking, Phys. Lett. B 564 (2003) 73 [hep-ph/0212002] [INSPIRE].

[26] M. Endo, K. Hamaguchi, S. Iwamoto and N. Yokozaki, Higgs mass, muon g- 2 and LHC prospects in gauge mediation models with vector-like matters, Phys. Rev. D 85 (2012) 095012 [arXiv:1112.5653] [INSPIRE].

[27] H.M. Tran and H.T. Nguyen, GUT-inspired MSSM in light of muon g- 2 and LHC results at $\sqrt{s}=13$ TeV, Phys. Rev. D 99 (2019) 035040 [arXiv:1812.11757] [INSPIRE].

[28] T. Fukuyama, N. Okada and H.M. Tran, Sparticle spectroscopy of the minimal SO(10) model, Phys. Lett. B 767 (2017) 295 [arXiv:1611.08341] [INSPIRE].

[29] P. Cox, C. Han and T.T. Yanagida, Muon $g-2$ and dark matter in the minimal supersymmetric standard model, Phys. Rev. D 98 (2018) 055015 [arXiv:1805.02802] [INSPIRE]. 
[30] A. Kobakhidze, M. Talia and L. Wu, Probing the MSSM explanation of the muon $g-2$ anomaly in dark matter experiments and at a $100 \mathrm{TeV}$ pp collider, Phys. Rev. D 95 (2017) 055023 [arXiv: 1608.03641] [INSPIRE].

[31] G.-C. Cho, K. Hagiwara, Y. Matsumoto and D. Nomura, The MSSM confronts the precision electroweak data and the muon $g-2$, JHEP 11 (2011) 068 [arXiv:1104.1769] [INSPIRE].

[32] M. Endo, K. Hamaguchi, S. Iwamoto and T. Yoshinaga, Muon g- 2 vs LHC in Supersymmetric Models, JHEP 01 (2014) 123 [arXiv:1303.4256] [INSPIRE].

[33] M.A. Ajaib, B. Dutta, T. Ghosh, I. Gogoladze and Q. Shafi, Neutralinos and sleptons at the LHC in light of muon $(g-2)_{\mu}$, Phys. Rev. D 92 (2015) 075033 [arXiv:1505. 05896] [INSPIRE].

[34] M. Badziak, Z. Lalak, M. Lewicki, M. Olechowski and S. Pokorski, Upper bounds on sparticle masses from muon $g-2$ and the Higgs mass and the complementarity of future colliders, JHEP 03 (2015) 003 [arXiv:1411.1450] [INSPIRE].

[35] D. Sabatta, A.S. Cornell, A. Goyal, M. Kumar, B. Mellado and X. Ruan, Connecting the Muon Anomalous Magnetic Moment and the Multi-lepton Anomalies at the LHC, arXiv: 1909.03969 [INSPIRE].

[36] B.P. Padley, K. Sinha and K. Wang, Natural Supersymmetry, Muon g-2 and the Last Crevices for the Top Squark, Phys. Rev. D 92 (2015) 055025 [arXiv:1505.05877] [InSPIRE].

[37] H.E. Haber and G.L. Kane, The Search for Supersymmetry: Probing Physics Beyond the Standard Model, Phys. Rept. 117 (1985) 75 [INSPIRE].

[38] N. Arkani-Hamed, A. Delgado and G.F. Giudice, The Well-tempered neutralino, Nucl. Phys. B $\mathbf{7 4 1}$ (2006) 108 [hep-ph/0601041] [INSPIRE].

[39] C. Han, A. Kobakhidze, N. Liu, A. Saavedra, L. Wu and J.M. Yang, Probing Light Higgsinos in Natural SUSY from Monojet Signals at the LHC, JHEP 02 (2014) 049 [arXiv:1310.4274] [INSPIRE].

[40] G. Pozzo and Y. Zhang, Constraining resonant dark matter with combined LHC electroweakino searches, Phys. Lett. B 789 (2019) 582 [arXiv:1807.01476] [InSPIRE].

[41] S. Profumo, T. Stefaniak and L. Stephenson Haskins, The Not-So-Well Tempered Neutralino, Phys. Rev. D 96 (2017) 055018 [arXiv:1706.08537] [InSPIRE].

[42] M. Abdughani, L. Wu and J.M. Yang, Status and prospects of light bino-higgsino dark matter in natural SUSY, Eur. Phys. J. C 78 (2018) 4 [arXiv: 1705.09164] [INSPIRE].

[43] M. Abdughani and L. Wu, On the coverage of neutralino dark matter in coannihilations at the upgraded $L H C$, arXiv:1908.11350 [INSPIRE].

[44] M. Endo, K. Hamaguchi, T. Kitahara and T. Yoshinaga, Probing Bino contribution to muon $g-2$, JHEP 11 (2013) 013 [arXiv:1309.3065] [INSPIRE].

[45] H. Fargnoli, C. Gnendiger, S. Paßehr, D. Stöckinger and H. Stöckinger-Kim, Two-loop corrections to the muon magnetic moment from fermion/sfermion loops in the MSSM: detailed results, JHEP 02 (2014) 070 [arXiv:1311.1775] [INSPIRE].

[46] H.G. Fargnoli, C. Gnendiger, S. Paßehr, D. Stöckinger and H. Stöckinger-Kim, Non-decoupling two-loop corrections to $(g-2)_{\mu}$ from fermion/sfermion loops in the MSSM, Phys. Lett. B 726 (2013) 717 [arXiv:1309.0980] [INSPIRE].

[47] A. Djouadi, M.M. Muhlleitner and M. Spira, Decays of supersymmetric particles: The Program SUSY-HIT (SUspect-SdecaY-HDECAY-InTerface), Acta Phys. Polon. B 38 (2007) 635 [hep-ph/0609292] [INSPIRE]. 
[48] D. Chowdhury, R.M. Godbole, K.A. Mohan and S.K. Vempati, Charge and Color Breaking Constraints in MSSM after the Higgs Discovery at LHC, JHEP 02 (2014) 110 [Erratum ibid. 03 (2018) 149] [arXiv: 1310.1932] [INSPIRE].

[49] G. Bélanger et al., Indirect search for dark matter with MicrOMEGAs2.4, Comput. Phys. Commun. 182 (2011) 842 [arXiv: 1004.1092] [INSPIRE].

[50] Planck collaboration, Planck 2015 results. XIII. Cosmological parameters, Astron. Astrophys. 594 (2016) A13 [arXiv:1502.01589] [INSPIRE].

[51] C. Han, L. Wu, J.M. Yang, M. Zhang and Y. Zhang, New approach for detecting a compressed bino/wino at the LHC, Phys. Rev. D 91 (2015) 055030 [arXiv:1409.4533] [INSPIRE].

[52] G.H. Duan, K.-I. Hikasa, J. Ren, L. Wu and J.M. Yang, Probing bino-wino coannihilation dark matter below the neutrino floor at the LHC, Phys. Rev. D 98 (2018) 015010 [arXiv: 1804.05238] [INSPIRE].

[53] LUX collaboration, Limits on spin-dependent WIMP-nucleon cross section obtained from the complete LUX exposure, Phys. Rev. Lett. 118 (2017) 251302 [arXiv:1705. 03380] [inSPIRE].

[54] XENON collaboration, First Dark Matter Search Results from the XENON1T Experiment, Phys. Rev. Lett. 119 (2017) 181301 [arXiv:1705. 06655] [INSPIRE].

[55] PandaX-II collaboration, Dark Matter Results From 54-Ton-Day Exposure of PandaX-II Experiment, Phys. Rev. Lett. 119 (2017) 181302 [arXiv:1708.06917] [INSPIRE].

[56] LZ collaboration, LUX-ZEPLIN (LZ) Conceptual Design Report, arXiv:1509.02910 [INSPIRE].

[57] ATLAS collaboration, Search for electroweak production of charginos and sleptons decaying into final states with two leptons and missing transverse momentum in $\sqrt{s}=13 \mathrm{TeV} p p$ collisions using the ATLAS detector, arXiv:1908.08215 [INSPIRE].

[58] ATLAS collaboration, Searches for electroweak production of supersymmetric particles with compressed mass spectra in $\sqrt{s}=13 \mathrm{TeV}$ pp collisions with the ATLAS detector, ATLAS-CONF-2019-014.

[59] J. Alwall et al., The automated computation of tree-level and next-to-leading order differential cross sections and their matching to parton shower simulations, JHEP 07 (2014) 079 [arXiv: 1405.0301] [INSPIRE].

[60] T. Sjöstrand et al., An Introduction to PYTHIA 8.2, Comput. Phys. Commun. 191 (2015) 159 [arXiv: 1410.3012] [INSPIRE].

[61] DELPHES 3 collaboration, DELPHES 3, A modular framework for fast simulation of a generic collider experiment, JHEP 02 (2014) 057 [arXiv: 1307.6346] [INSPIRE].

[62] M. Drees, H. Dreiner, D. Schmeier, J. Tattersall and J.S. Kim, CheckMATE: Confronting your Favourite New Physics Model with LHC Data, Comput. Phys. Commun. 187 (2015) 227 [arXiv: 1312.2591] [INSPIRE].

[63] J.S. Kim, D. Schmeier, J. Tattersall and K. Rolbiecki, A framework to create customised LHC analyses within CheckMATE, Comput. Phys. Commun. 196 (2015) 535 [arXiv: 1503.01123] [INSPIRE].

[64] D. Dercks, N. Desai, J.S. Kim, K. Rolbiecki, J. Tattersall and T. Weber, CheckMATE 2: From the model to the limit, Comput. Phys. Commun. 221 (2017) 383 [arXiv:1611.09856] [INSPIRE]. 
[65] C.G. Lester and D.J. Summers, Measuring masses of semiinvisibly decaying particles pair produced at hadron colliders, Phys. Lett. B 463 (1999) 99 [hep-ph/9906349] [INSPIRE].

[66] A. Barr, C. Lester and P. Stephens, A variable for measuring masses at hadron colliders when missing energy is expected; $m_{T 2}$ : the Truth behind the glamour, J. Phys. G 29 (2003) 2343 [hep-ph/0304226] [INSPIRE].

[67] C. Lester and A. Barr, MTGEN: Mass scale measurements in pair-production at colliders, JHEP 12 (2007) 102 [arXiv:0708.1028] [INSPIRE].

[68] K. Albertsson et al., Machine Learning in High Energy Physics Community White Paper, J. Phys. Conf. Ser. 1085 (2018) 022008 [arXiv: 1807.02876] [InSPIRE].

[69] M. Abdughani, J. Ren, L. Wu, J.M. Yang and J. Zhao, Supervised deep learning in high energy phenomenology: a mini review, Commun. Theor. Phys. 71 (2019) 955 [arXiv: 1905.06047] [INSPIRE].

[70] M. Abdughani, J. Ren, L. Wu and J.M. Yang, Probing stop pair production at the LHC with graph neural networks, JHEP 08 (2019) 055 [arXiv: 1807.09088] [INSPIRE].

[71] J. Ren, L. Wu, J.M. Yang and J. Zhao, Exploring supersymmetry with machine learning, Nucl. Phys. B 943 (2019) 114613 [arXiv:1708.06615] [INSPIRE].

[72] S. Caron, J.S. Kim, K. Rolbiecki, R. Ruiz de Austri and B. Stienen, The BSM-AI project: SUSY-AI-generalizing LHC limits on supersymmetry with machine learning, Eur. Phys. J. C 77 (2017) 257 [arXiv: 1605. 02797] [inSPIRE].

[73] S. Dawson et al., Working Group Report: Higgs Boson, in Proceedings, 2013 Community Summer Study on the Future of U.S. Particle Physics: Snowmass on the Mississippi (CSS2013), Minneapolis, MN, U.S.A., 29 July-6 August 2013 (2013) [arXiv:1310.8361] [INSPIRE].

[74] T. Barklow et al., ILC Operating Scenarios, arXiv:1506.07830 [INSPIRE].

[75] TleP Design Study Working Group collaboration, First Look at the Physics Case of TLEP, JHEP 01 (2014) 164 [arXiv:1308.6176] [INSPIRE].

[76] CEPC Study Group collaboration, CEPC Conceptual Design Report: Volume $2-$ Physics \& Detector, arXiv:1811.10545 [INSPIRE].

[77] J. Cao, L. Wu, P. Wu and J.M. Yang, The Z+photon and diphoton decays of the Higgs boson as a joint probe of low energy SUSY models, JHEP 09 (2013) 043 [arXiv:1301.4641] [INSPIRE].

[78] S.L. Hu, N. Liu, J. Ren and L. Wu, Revisiting Associated Production of $125 \mathrm{GeV}$ Higgs Boson with a Photon at a Higgs Factory, J. Phys. G 41 (2014) 125004 [arXiv:1402.3050] [INSPIRE].

[79] J. Cao, C. Han, J. Ren, L. Wu, J.M. Yang and Y. Zhang, SUSY effects in Higgs productions at high energy $e^{+} e^{-}$colliders, Chin. Phys. C 40 (2016) 113104 [arXiv:1410.1018] [INSPIRE].

[80] A. Kobakhidze, N. Liu, L. Wu and J. Yue, Implications of CP-violating Top-Higgs Couplings at LHC and Higgs Factories, Phys. Rev. D 95 (2017) 015016 [arXiv:1610.06676] [INSPIRE].

[81] L. Wu, H. Zhang and B. Zhu, Non-minimal Higgs-gravity coupling and naturalness at the LHC and beyond, JCAP 07 (2019) 033 [arXiv:1901.06532] [INSPIRE].

[82] C. Han, A. Kobakhidze, N. Liu, L. Wu and B. Yang, Constraining Top partner and Naturalness at the LHC and TLEP, Nucl. Phys. B 890 (2014) 388 [arXiv:1405.1498] [INSPIRE]. 
[83] L. Wu, J.M. Yang, C.-P. Yuan and M. Zhang, Higgs self-coupling in the MSSM and NMSSM after the LHC Run 1, Phys. Lett. B 747 (2015) 378 [arXiv: 1504.06932] [INSPIRE].

[84] M. Carena, J.R. Espinosa, M. Quirós and C.E.M. Wagner, Analytical expressions for radiatively corrected Higgs masses and couplings in the MSSM, Phys. Lett. B 355 (1995) 209 [hep-ph/9504316] [INSPIRE].

[85] S. Heinemeyer, W. Hollik and G. Weiglein, FeynHiggs: A Program for the calculation of the masses of the neutral CP even Higgs bosons in the MSSM, Comput. Phys. Commun. 124 (2000) 76 [hep-ph/9812320] [INSPIRE]. 